\title{
One Step Production of Bicomponent Yarns with Glass Fibre Core and Thermoplastic Sheath for Composite Applications
}

\author{
Alexander Lüking ${ }^{1}$, Robert Brüll ${ }^{1}$, Thomas Köhler ${ }^{1^{*}}$, Davide Pico, \\ Gunnar Seide ${ }^{1,2}$, Thomas Gries ${ }^{1}$ \\ ${ }^{1}$ Institute for Textile Technology of RWTH Aachen University, Aachen, Germany \\ ${ }^{2}$ Aachen-Maastricht Institute for Biobased Materials, Geleen, Netherlands \\ *thomas.köhler@ita.rwth-aachen.de
}

Keywords: Fibre reinforced thermoplastic composites, Glass fibre, coating, PA 12, melt spinning

\begin{abstract}
The film stacking method is the industrial standard for the manufacturing of fibre reinforced thermoplastic composites (FRTCs). An alternative to this is commingling thermoplastic fibres with reinforcement fibres, e. g. glass fibres, into hybrid yarns. However, the composites produced by the use of film-stacking or hybrid yarns cannot achieve an optimal impregnation of reinforcement fibres with the matrix polymer. This stems from the high melt viscosity of thermoplastics, which prevents a uniform wetting of the reinforcement fibres. Leaving some fibers unconnected to the matrix. This leads to a composite lower strength than possible in theory. The aim of the research is the coating of a single glass filament in the glass fibre nozzle drawing process to achieve a homogenous distribution of glass fibres and matrix in the final composite. The approach uses particles with a diameter from 5 to $25 \mu \mathrm{m}$ of polyamide 12 (PA12) which are electrostatically charged and blown at an E-glass filament in the nozzle drawing process. The particles adhering to the filament are melted by infrared heating and winded afterwards. This process allows for a homogenous distribution of fibres and matrix in the thermoplastic composite and therefore leads to a higher fibre volume content which improves mechanical properties. Even though the glass filaments could be coated with PA12, a homogenous sheath could not be achieved in this investigation. Therefore, further research will focus on an improved homogeneity by reducing the agglomeration of PA12, using dried PA12 and enhancing the coating setup.
\end{abstract}

\section{Introduction}

Fibre reinforced plastics (FRPs) are used in different industrial areas such aerospace, automotive and sports equipment industry. In the production of FRPs, fibres are combined with a matrix material in order to create a new material which combines the advantages of both parts [1]. Typical fibres used in FRPs are glass, carbon or aramid. They can be combined with thermoplastic or thermosetting matrices. Typical thermoplastics are polyamide (PA), polypropylene (PP) or polyethylene (PE). In conventional production processes like resign transfer moulding (RTM), fibres and matrix are combined in the final step of component production [2]. To simplify this process prepregs are used. Prepregs are already impregnated fibres or fibre structures which can be assembled to a FRP component. Common methods for the production of prepregs are the impregnation of the reinforcement fibres with the melted or the solved matrix or the commingling of matrix and reinforcement fibres. In the melted impregnation the matrix material is melted and the fibres are then impregnated with the liquid matrix. This is only possible for thermoplastic materials. For solvent impregnation a dissolved matrix is used. The matrix solution needs a low viscosity to be applied onto the fibres [3]. The commingling process combines reinforcement with matrix fibres. Here, an air jet nozzle is used to mix the fibres and produce a hybrid yarn [4]. All kinds of thermoplastic prepregs can then be consolidated through compression moulding. A disadvantage of all the common processes for the production of prepregs is the extra step needed to combine fibres with matrix material. Thus, the price for the products is increased [5,6]. Moreover, an important characteristic for composites is the volume ratio between fibre and matrix. The specific strength and stiffness of the component is mostly defined by this ratio [1,2]. Hence through an adjustment of the fibre volume content the strength and stiffness of the component can be customized. A specific 
variation of the fibre volume ratio is not possible with the mentioned prepreg processes. Within this paper a process is shown, that makes an integration of the fibre impregnation into the glass fibre spinning process possible. With this process it will be possible to adjust the fibre volume content for customized FRCTs and to get a shorter production chain.

\section{Methods}

In this study a process is determined that introduces the coating of glass fibres with PA12 into the glass fibre spinning process. For this purpose, a powder of PA12 particles is blown onto the glass fibres with a corona gun. The powder is then melted and the glass filament is winded. The process is shown in Fig. 1. In the following the components and processes that lead to a coated fibre are shown.

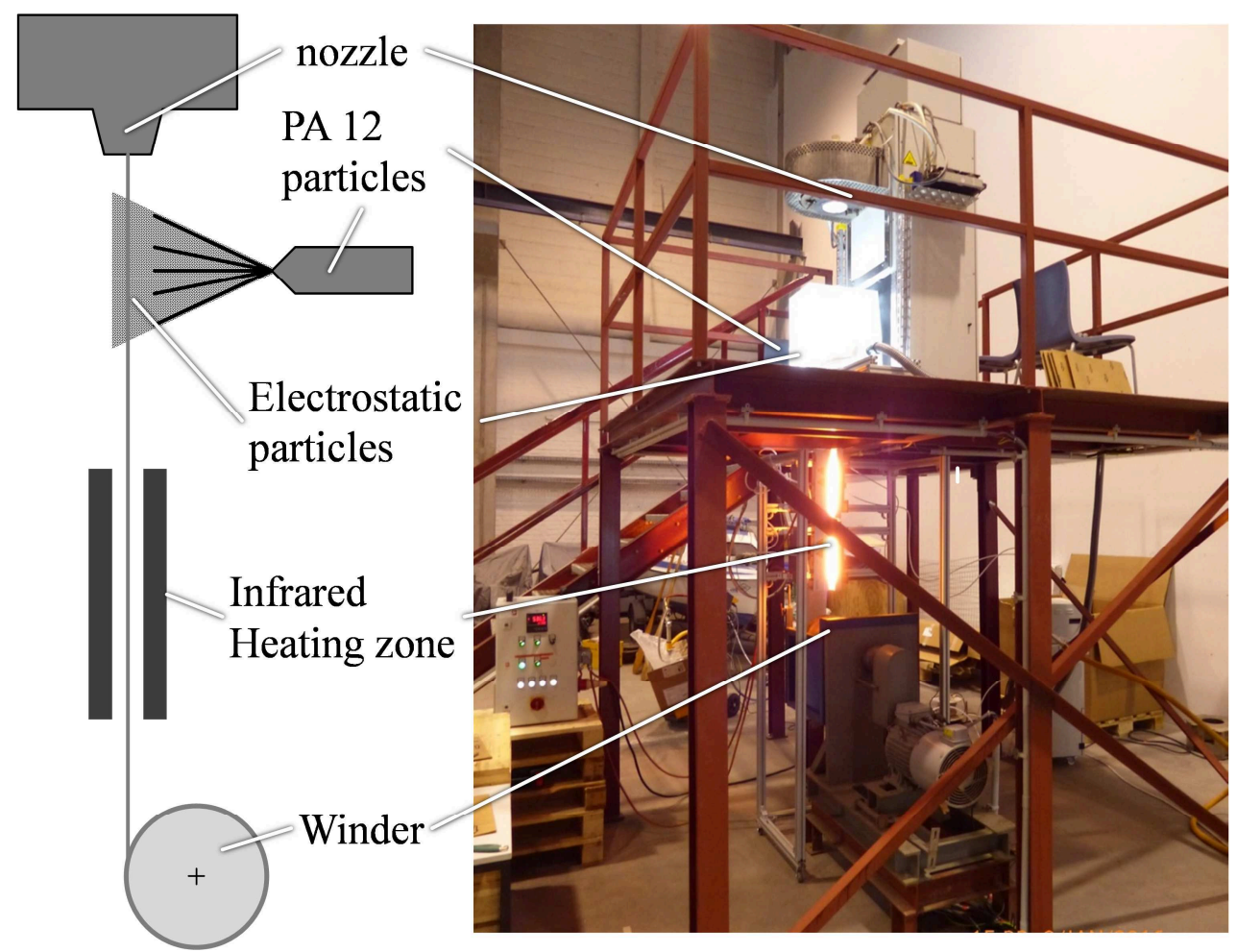

Figure 1: Process for coating during the glass spinning process of one single filament

\subsection{Spinning of the glass fibres}

For spinning, the E-glass filament granulate is heated up to $1.250{ }^{\circ} \mathrm{C}$. The melt flows through the nozzle so that the fibres are formed. The glass freezes and the fibres are winded up. The winding speed defines the diameter of the formed fibres. For the coating experiment a winding speed of $50 \mathrm{~m} / \mathrm{min}$ is used.

\subsection{Powder application with corona gun}

For a thin coating PA12 particles with a diameter of 5 to $25 \mu \mathrm{m}$ are used. For this the PA12 powder VESTOSINT $^{\circledR}$ by Evonik Industries AG, Essen/Germany is chosen. To ensure the adherence between fibre and powder an electrostatic charging of the particles is necessary. For this charging, a gun using the corona method is used. In this method, the particles are charged by passing a high voltage cascade inside the gun. The particles are then ionized and adhere to the fibres. For these experiments OptiSelect Handgun from Gema Switzerland GmbH, St. Gallen/Switzerland is used. It has a control unit which regulates the powder/air ratio. The powder is stored inside a tank that is positioned on a vibrating plate. Into the powder the suction tube is stuck, which is also connected to the pistol. The vibration of the plate ensures that there is constantly powder flowing to the gun. The experiments are executed with $50 \%$ and $100 \%$ powder/air ratio. Additionally, the application of more powder by the principle of contact is tested. Therefore, the fibres are lead through powder on two rollers. The principle is shown in Fig. 2. 


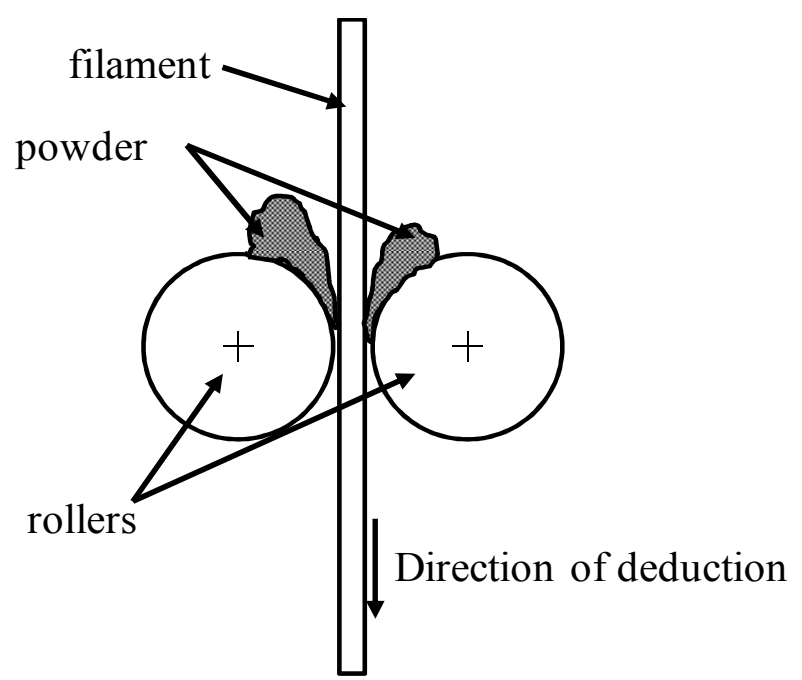

Figure 2: Principle of applying more powder through contact

\subsection{Melting of the powder}

To fix the powder on the glass fibres it is necessary to melt the powder onto the surface. Therefor a heating passage is added behind the powder application. Two infrared heaters of the type lineIR 360 $C P$ from Mehler Engineering + Service GmbH, Fulda/Germany are arranged in series. The heating coil is arranged inside a quartz tube filled with inert gas. Because of this the coil can reach higher temperatures. Facing the heating elements two reflectors are installed which make sure that there is a heat spread throughout the whole fibres. The heaters are connected to compressed air which can cool them down. A schematic sketch of the heater is shown in Fig. 3.

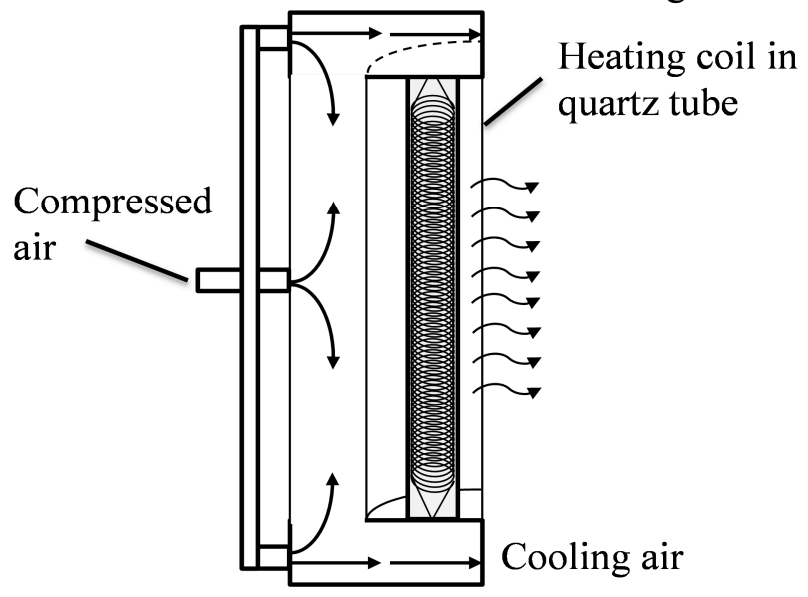

Figure 3: Schematic sketch of the heater

\subsection{Analysis of the coated fibres}

The fibres are analysed by using the scanning electron microscope Leo 1450 VP from Carl Zeiss $A G$, Oberkochen/Germany. The fibres are fixed onto a metal plate by using carbon tapes. A layer of electro conductive gold prevents the sample from electrical charging through the electron beam of the microscope. To get a sectional view of the coated fibres the samples are fixed between to metal plates and cut with a razor blade.

\section{Results}

The integration of the coating into the spinning process has no negative impact on the spinning process. The filaments are not deflected from their original path. However, the cooling air inside the heaters sets the fibres into oscillation. For analysing the coating success, the produced fibres are observed with the scanning electron microscope. In Figure 4 a glass filament with PA12 powder particles on its surface is shown. This filament was not guided through the heaters. Because of this, the particles are not melted. This figure shows that the particles adhere to the filament. The filament diameter is about $40 \mu \mathrm{m}$. 


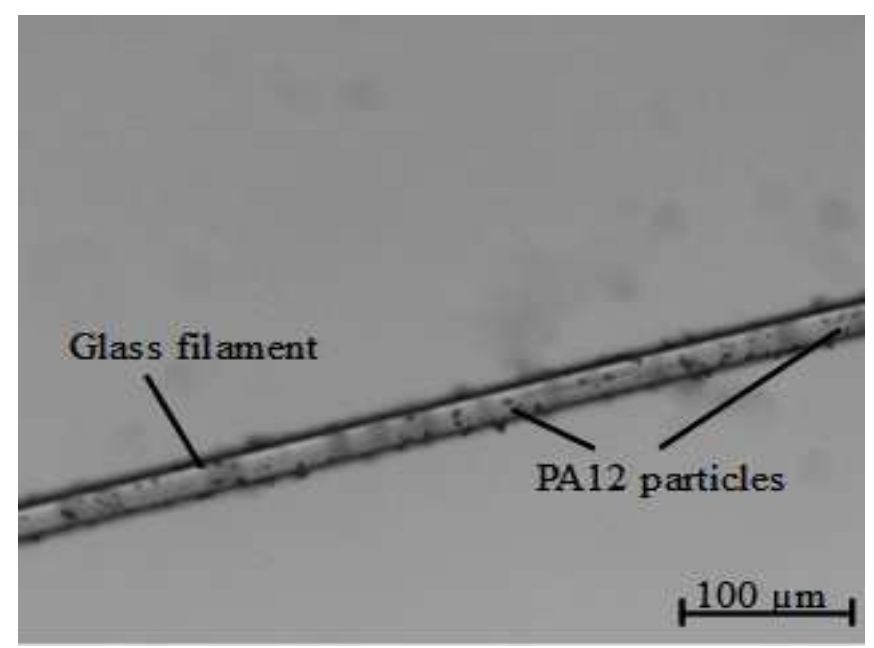

Figure 4: Glass filament with PA12 particles

When the filament is guided through the heating area, the particles melt and they build a film on the filament surface. Figure 5 shows fibres produced with a particle/air ratio of $50 \%$ and $100 \%$.
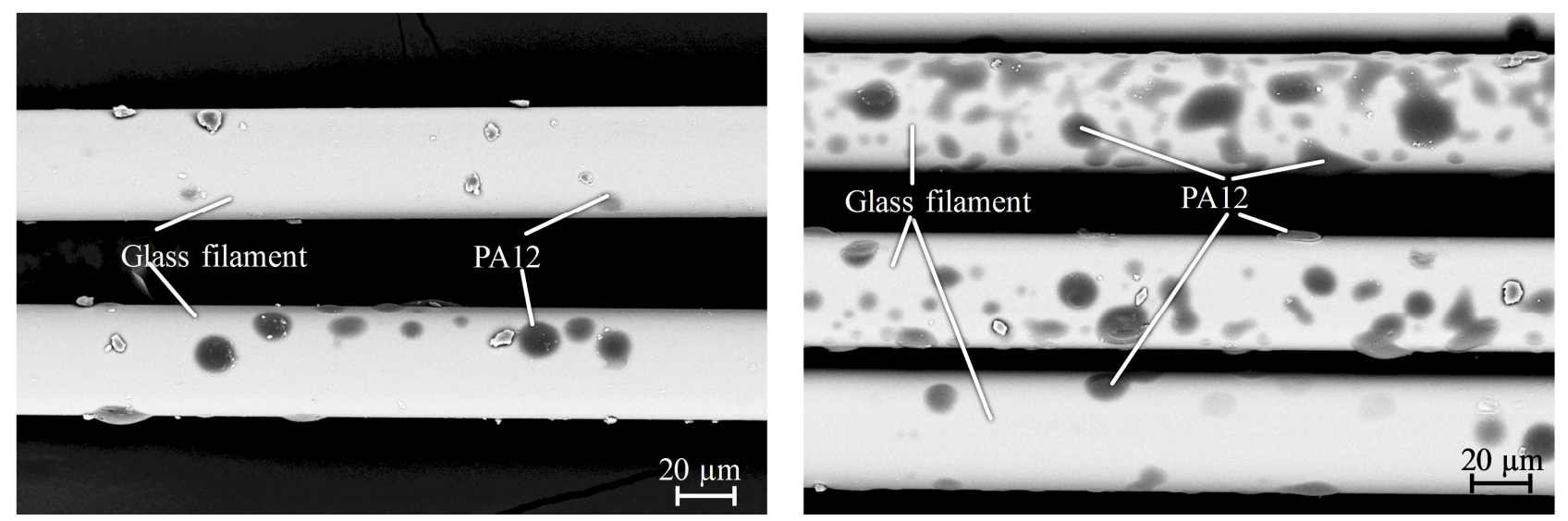

Figure 5: Glass filaments coated with PA12 particles. Particle/air ratio $50 \%$ (left) and particle/air ratio $100 \%$ (right)

The filaments produced with the $50 \%$ ratio show diverse amount of PA12 areas. The bottom filament shows more coated areas than the top one. The filaments produced with the $100 \%$ ratio have more particles on their surfaces but still differ in the amount of PA12 although they have all been produced with the same process adjustments. On the top filament approx. $40 \%$ of its surface is covered with PA12 whereas only $15 \%$ of the bottom filaments' surface is covered. In Figure 6 filaments are shown where additionally to the powder gun, particles are applied by contact with two rollers. Approximately $25 \%$ of the surface is covered with PA12 particles. These filaments show more particles than without the contact coating but less than the filaments with $100 \%$ ratio.

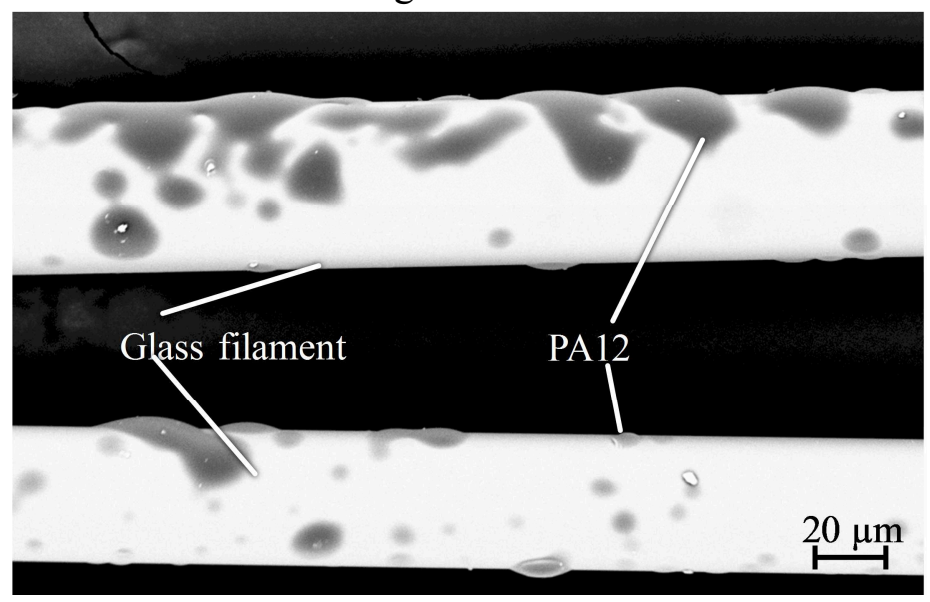

Figure 6: Filaments coated with $50 \%$ ratio and additional contact coating 


\section{Discussion}

It has been shown that it is possible to coat glass filaments with powder during the spinning process. The electro static charging supports the adhesion of the particles onto the filaments. Moreover, it has been shown that it is possible to melt the powder by passing the heating area. The produced filament diameter varies between 35 and $45 \mu \mathrm{m}$ which is a lot higher than the intended $15 \mu \mathrm{m}$. This result is due to the low winding speed used during the experiments.

The microscopic pictures demonstrate that the number of particles on the filament's surfaces differ depending on parameters and setup. At first, these differences can be explained by different process settings. Using a particle/air ratio of $100 \%$ leads to a higher number of particles on the filament than using the $50 \%$ ratio does. The higher the ratio the more particles adhere on the surface. Apparently, the amount of PA12 can be controlled by adjusting the ratio of the powder gun. The additional contact coating does not lead to the desired result. There are less particles on the filament's surface than in the experiment with $100 \%$ ratio. One possible explanation is the missing electro static charging of the powder. Because of this it may be possible that the particles are shaken off the glass surface before they could be fixed inside the heating area.

Moreover, differences between filaments produced with the same settings can be observed. Miscellaneous reasons for this can be found. Firstly, an irregular powder emission of the powder gun can lead to an uneven amount of PA12 on the glass surface. Secondly, the kind of application can cause problems. Hence the powder is applied from one side the powder will more likely stick on this side rather than on the opposite side. Another reason for the irregularities can be found in the cooling air of the heaters. The air sets the filament into oscillation which can lead to a removal of particles before melting.

The figures show that there is no impregnation of the whole filament surface because not enough powder was fixed onto the surface. There are two possible explanations for this. Firstly, not enough of the powder adhered on the glass surface. Secondly, already adhered powder lost contact.

The experiments show, that coating glass filaments with PA12 particles is possible. However, it is not possible to draw conclusions for higher winding speed. The normal glass spinning speed is ten times higher than the speed used in these experiments. To achieve the same results with higher winding speeds, higher attraction forces between powder and filaments are necessary. Moreover, the duration inside the heating areas would decrease rapidly. Thus, it is uncertain if the heating time is long enough to meld the particles.

\section{Conclusion}

In this study it is shown that a coating of glass filaments during the spinning process is possible. The powder is melted during the spinning process and a regular coating can be achieved. The adhesion between powder and fibre is high enough for the composite fibres to be handled afterwards, without peeling it off. However, diameters of $35 \mu \mathrm{m}$ were achieved. Further experiments with higher production speed, up to $1.000 \mathrm{~m} / \mathrm{min}$, are essential for this technology, because the mechanical properties of the glass filament will increase. Additionally other thermoplastic particles (e. g. PP or PE) will be used. The contact coating is also considered more intense because a great potential is apparent. 


\section{References}

[1] W. Michaeli, M. Wegener, Einführung in die Technologie der Faserverbundwerkstoffe. Hanser, München, Wien, 1998.

[2] M. Neitzel, P. Mitschang, Handbuch der Verbundwerkstoffe: Werkstoffe, Verarbeitung, Anwendung, Hanser, München, Wien, 2004.

[3] R. Stolze, Imprägniertechniken für aramid- und kohlenstofffaserverstärkte Thermoplaste, Kunststoffe. 78 / 2 (1988) 126-131.

[4] B.-D. Choi, Entwicklung von Commingling-Hybridgarnen für langfaserverstärkte thermoplastische Verbundwerkstoffe, TUDpress, Verl. Der Wiss., Dresden, 2005.

[5] A. Lutz, Beitrag zur Entwicklung innovativer Fertigungstechniken für die Verarbeitung thermoplastischer Faserverbundwerkstoffe. Dissertation, Institut für Verbundwerkstoffe GmbH, Universität Kaiserslautern, Kaiserslautern, 1999.

[6] K. Henning, T. Gries, Wirtschaftliche Herstellung von Faserverbundbauteilen mit Hilfe automatisiert hergestellter textiler Preforms, in: Unternehmenskybernetik in der Praxis (14), Shaker, Aachen, 2008. 